\title{
TRYING A FERTIGATION PROGRAM FOR BEAN USING EXPERT SYSTEM

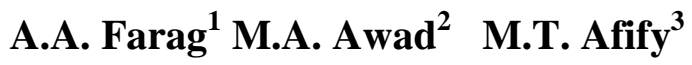 \\ ABSTRACT
}

The objective of this study is to design a fertigation program for bean (Phaseolus vulgaris L.) using the expert system (ES).To achieve the objective of this study, the following steps were required: $i$. identification of the problem ii. analysis of the information iii. characterizing the variables of the key factors and qualifiers. The study involved also a comparison between the ES program outputs and the corresponding ones recommended by the Ministry of Agriculture. To establish such a comparative study, a field experiment was executed on bean plant after dividing the filed of study into two sections. In the first, the experimental work was carried out using the ES fertigation management, while in the second section, the well known "CROPWAT Program", was used for the scheduling of the irrigation together with the traditional methods of fertigation outlined by the Ministry of Agriculture, Egypt. The results of comparison assured the superiority of the ES over the other traditional one, where higher values of water use efficiency "WUE" and nutrient use efficiency "NUE" were achived by the former than the latter.

Keywords: expert system, fertigation, irrigation, scheduling, WUE, NUE.

\section{INTRODUCTION}

Tertigation is a technique of fertilizer application through the water
of irrigation. With the use of modern water saving irrigation
systems such as drip and sprinkler systems, fertigation will be a promising technique. There are some advantages of fertigation which include easy application, use in adverse factors, low hazards, conservation of proper soil structure, possible control of pests and weeds and decreasing the adverse effect of salinity. However, the disadvantages of this system include increases in capital expenditure, incidents of orifices clogging, incidents of salinity build-up and need for technical handling (Charles, 2007).

1; 2; 3 Teaching Assist, Assoc. Prof. and .lecuterer, Ag. Eng. Dept., Fac. Ag., Benha Univ.

Misr. J. Ag. Eng., Jan 2012 
The agricultural sector in Egypt consumes about more than 81 percent from total available water and about 1.25 million tons of fertilizer annually (FAO, 2005). This problem forces the scientists to find out a new technique to overcome reasons of such problem. One of these techniques is using the fertigation system to increase the efficiency of both fertilization and irrigation.

The expert system (ES) is a computer program designed to simulate the problem solving behavior of an expert in a narrow domain or discipline (Rafea, 1998). The advantages of ES programs are minimizing or avoiding errors in complex tasks, protecting the perishable knowledge of experts and make it available and where required, systematically considering all possible alternatives, displaying unbiased judgment, available for use unlike human experts and less expensive to consult than human experts (Kabany, 2003, Awady, 2010 and Dent et al., 1989).

There are several problems associated with using the fertigation in terms of its management, these are lack in the efficiency of fertilizer and water management in the combined system of fertigation, fail in the finding of the best source of nutrients, optimum rates of fertilization, optimum rates of water, suitable timing, proper fertilizer placement and there is no particular system available to control fertigation technique under different conditions (Charles, 2007).

The objective of this research is to design an expert system to provide farmers by the sound decisions on the management of irrigation and fertilization (fertigation). There are also some specific objectives of this study which can be summarized in the following:

1 - Improving the efficiency of fertilizer and water use.

2 - Finding best sources of nutrients, optimum rates of fertilization, optimum water requirement, suitable timing and proper of fertilizer placement.

\section{MATRIALS AND METHODS}

To design the fertigation expert system program, we used the following materials:

- Microsoft visual C\#.net 2005

- Microsoft Access 2003

- Pc. Pentium 4. 


\section{Building the expert system}

The following steps were conducted for designing the expert system:

\section{a. Identification of the problem}

The problem of this study is to find out a new technique for solving the problems associated with fertigation system.

\section{b. Conceptualization}

This process involves the information analysis and identifying the decision making process and activities related to the application priorities of fertigation under different farm systems.

\section{c. Formulation}

Formulation involves characterizing the variables; the key factors and qualifiers for fertigation technique under diverse farm situation and conditions. Also, this procedure involves the representation of the variables; key factors and qualifiers into the production rules that make it usable within the development environment of the construction of the expert system rule-based program. Easiest and best ways to represent knowledge and data analysis is the development of knowledge and data as rules.

\section{d. Implementation}

We designed a computer program to represent and analyses fertigation data by using Visual C\#.net language.

\section{e. Verification:}

We compared the program ES output with the well-known "CROPWAT Program" used for the scheduling of the irrigation together with the traditional methods of fertigation outlined by the Ministry of Agriculture, Egypt.

\section{f. Validation of the expert system:}

We used fertigation program to manage the experimental management on bean in the Farm of the Faculty of Agriculture at Moshtohor.

\section{Inference Knowledge}

The design of inference knowledge consists of two main parts namely: inference structure and inference specification. The following paragraphs explain them. 


\section{Inference Structure}

As shown in the following the inference structure includes six inference steps. The objective of the expand inference is to use known data to derive new ones using a set of relations that forms the expansion model. The goal of Et0 irrigation schedule is to use the evapotranspiration (Et0) model. The goal of EtCrop is to use the model to generate an EtCrop. The goal of Water requirement is to generate a Water requirement more details.the goal of fertilization model is to get the results of concentration of fertilizer in irrigation water.

\section{Input data of the expert system}

\section{a. Soil data}

Soil texture is clay loam, soil test type is Colwell $\mathrm{P}(\mathrm{mg} / \mathrm{kg})$, soil type is medium, critical phosphorus is $35 \mathrm{mg} \cdot \mathrm{kg}^{-1}$, critical potash is $130 \mathrm{mg} \cdot \mathrm{kg}^{-1}$, critical sulfate is $7.5 \mathrm{mg} \cdot \mathrm{kg}^{-1}$, soil field capacity is $36.8 \%$, soil weilting point is $17.4 \%$, bulk denisity is $1.4, \mathrm{EC}$ is $1.7 \mathrm{dS} \mathrm{m}^{-1}, \mathrm{pH}$ is 8.05 , calcium carbonate is $10 \%$, depletion ratio is $50 \%$, soil nitrogen content is 0.11 mg. $\mathrm{kg}^{-1}$, soil phosphorus content is $41.1 \mathrm{mg} \cdot \mathrm{kg}^{-1}$, soil potash content is $389.7 \mathrm{mg} \cdot \mathrm{kg}^{-1}$ and $\mathrm{C} / \mathrm{N}$ ratio is $37.2 / 1$.

\section{b. Climate data}

The climate data of Kaliobia Governorate are shown in Table (1).

Table (1): Climate data of Kaliobia Governorate, average values for the period extending form 1997 until 2006

\begin{tabular}{|c|c|c|c|c|c|c|c|}
\hline Month & $\begin{array}{c}\text { Extra } \\
\text { radiation }\end{array}$ & $\begin{array}{c}\text { Mean } \\
\text { relative } \\
\text { humidity }\end{array}$ & $\begin{array}{c}\text { Mean daily } \\
\text { actual sun- } \\
\text { shine hours }\end{array}$ & $\begin{array}{c}\text { Mean daily } \\
\text { max. } \\
\text { Sunshine } \\
\text { hours }\end{array}$ & $\begin{array}{c}\text { Max. } \\
\text { temp. }\end{array}$ & $\begin{array}{c}\text { Min. } \\
\text { temp. }\end{array}$ & $\begin{array}{c}\text { Average } \\
\text { temp. }\end{array}$ \\
\hline 1 & 8 & 60.58 & 12 & 8 & 19.7 & 8.9 & 12.35 \\
\hline 2 & 9 & 59.02 & 12 & 8 & 20 & 8.5 & 13.1 \\
\hline 3 & 13 & 61.60 & 12 & 9 & 22.8 & 10 & 15.25 \\
\hline 4 & 15 & 57.96 & 12 & 8 & 28.3 & 13.6 & 18.85 \\
\hline 5 & 16 & 52.37 & 10 & 8 & 33 & 17.1 & 23.05 \\
\hline 6 & 17 & 56.02 & 10 & 9 & 32.9 & 19.7 & 25.6 \\
\hline 7 & 17 & 59.81 & 10 & 9 & 35 & 22 & 26.85 \\
\hline 8 & 16 & 62.72 & 10 & 9 & 35.2 & 22.2 & 26.85 \\
\hline 9 & 14 & 57.17 & 10 & 7 & 32.6 & 20.2 & 24.9 \\
\hline 10 & 12 & 56.24 & 10 & 7 & 30.4 & 18.5 & 22.75 \\
\hline 11 & 10 & 55.01 & 11 & 8 & 25.7 & 14 & 19.35 \\
\hline 12 & 8 & 58.70 & 11 & 8 & 21.2 & 11.1 & 15.1 \\
\hline
\end{tabular}




\section{c. Water data}

Water source was analysed and the results of analyses are presented in Table (2):

Table (2): The properties of the used irrigation water

\begin{tabular}{|c|c|c|}
\hline Property & Unit & Value \\
\hline Electrical conductivity & $\mathrm{dS} / \mathrm{m}$ & 1.5 \\
\hline $\mathrm{pH}$ & & 7.3 \\
\hline Total Nitrogen & $\%$ & 0.001 \\
\hline $\mathrm{Na}^{+}$ & $\mathrm{mg} . \mathrm{L}^{-1}$ & 219 \\
\hline $\mathrm{Cl}^{-}$ & $\mathrm{mg} \cdot \mathrm{L}^{-1}$ & 418 \\
\hline $\mathrm{Mg}^{2+}$ & $\mathrm{mg} \cdot \mathrm{L}^{-1}$ & 0.48 \\
\hline
\end{tabular}

\section{d. Fertilizer data}

For the bean crop, the following fertilizers were used

Table (3): The fertizers used for the bean crop

\begin{tabular}{|l|c|c|c|c|c|}
\hline Fertilizer & State & $\mathbf{N \%}$ & $\begin{array}{c}\mathbf{P}_{\mathbf{2}} \mathbf{O}_{\mathbf{5}} \\
\mathbf{\%}\end{array}$ & $\begin{array}{c}\mathbf{K}_{\mathbf{2}} \mathbf{O} \\
\mathbf{\%}\end{array}$ & $\begin{array}{c}\mathbf{S} \\
\mathbf{\%}\end{array}$ \\
\hline Ammonium nitrate & Solid & 34 & 0 & 0 & 0 \\
\hline Phosphoric acid & liquid & 0 & 85 & 0 & 0 \\
\hline Potassium sulfate & Solid & 0 & 0 & 50 & 18 \\
\hline
\end{tabular}

\section{e. Crop data}

The bean crop data were: Crop name $=$ bean, Plant age $=110$ days, Plant height $=40 \mathrm{~cm}$, Root depth $=60 \mathrm{~cm}$, Intial stage $=20$ days, Develpement stage $=30$ days, Middle stage $=40$ days, Late stage $=20$ days, Depletion $=45 \%$, Nitrogen requirement $=40 \mathrm{~kg} /$ fed., $\mathrm{P}_{2} \mathrm{O}_{5}$ requirement $=48 \mathrm{~kg} /$ fed., $\mathrm{K}_{2} \mathrm{O}$ requirement $=48 \mathrm{~kg} /$ fed., $\mathrm{Kc}_{\text {intial }}=40$, $\mathrm{Kc}_{\text {mid }}=115$ and $\mathrm{Kc}_{\text {late }}=35$.

\section{f. Irrigation system data}

Three irrigation systems (sub-drip irrigation, drip irrigation and furrow irrigation) were used. The pump discharge was $5 \mathrm{~m}^{3} / \mathrm{h}$.

\section{- Data of the drip irrigation system and sub drip irrigation system:}

Injection device type $=$ Differential Tank, Pump discharge $=5 \mathrm{~m}^{3} / \mathrm{h}$, Efficiency $=90 \%$, wilting area $=35 \%$ and volume of fertilizer tank $=$ $0.4 \mathrm{~m}^{3}$ 
- Data of the surface irrigation (Furrow irrigation):

Injection device type $=$ Differential tank, Pump discharge $=5 \mathrm{~m}^{3} / \mathrm{h}$, Efficiency $=60 \%$, Wilting area $=100 \%$ and Volume of fertilizer tank $=$ $0.4 \mathrm{~m}^{3}$

\section{g. Data of crop tolerance}

$$
\mathrm{EC}_{\mathrm{e}} 100 \%=1, \mathrm{EC}_{\mathrm{e}} 90 \%=1.5, \mathrm{EC}_{\mathrm{e}} 75 \%=2.2, \mathrm{EC}_{\mathrm{e}} 50 \%=3.9 \text {, }
$$

$\mathrm{EC}_{\mathrm{e}} 0 \%=6.3, \mathrm{EC}_{\mathrm{w}} 100 \%=0.7, \mathrm{EC}_{\mathrm{w}} 90 \%=1, \mathrm{EC}_{\mathrm{w}} 75 \%=1.5, \mathrm{EC}_{\mathrm{w}}$ $50 \%=2.4, \mathrm{EC}_{\mathrm{w}} 0 \%=4.2$.

\section{h. Farm data}

Field study was applied in the Farm of the Faculty of Agriculture, Moshtohor, Tokh, Kaliobia, Egypt from March1, 2009 to June 19, 2009. The input farm data were area $=225 \mathrm{~m}^{2}$, farm latitude $=30^{0} 21^{\backslash} 21^{\prime \prime}$, farm longitude $=31^{0} 13^{\prime} 8^{\prime \prime}$, previous crop = other, crop type = summer crop, used pre-fertilization $=$ no, calcium carbonate $>=10 \%$, Manure use $=$ no, farm type $=$ open field and pump discharge $=5 \mathrm{~m}^{3} / \mathrm{h}$.

\section{3- Measurements}

The following parameters were determined under field and laboratory conditions. The farm was divided into six plots to study the effects of expert system management on the WUE and NUE for bean crop.

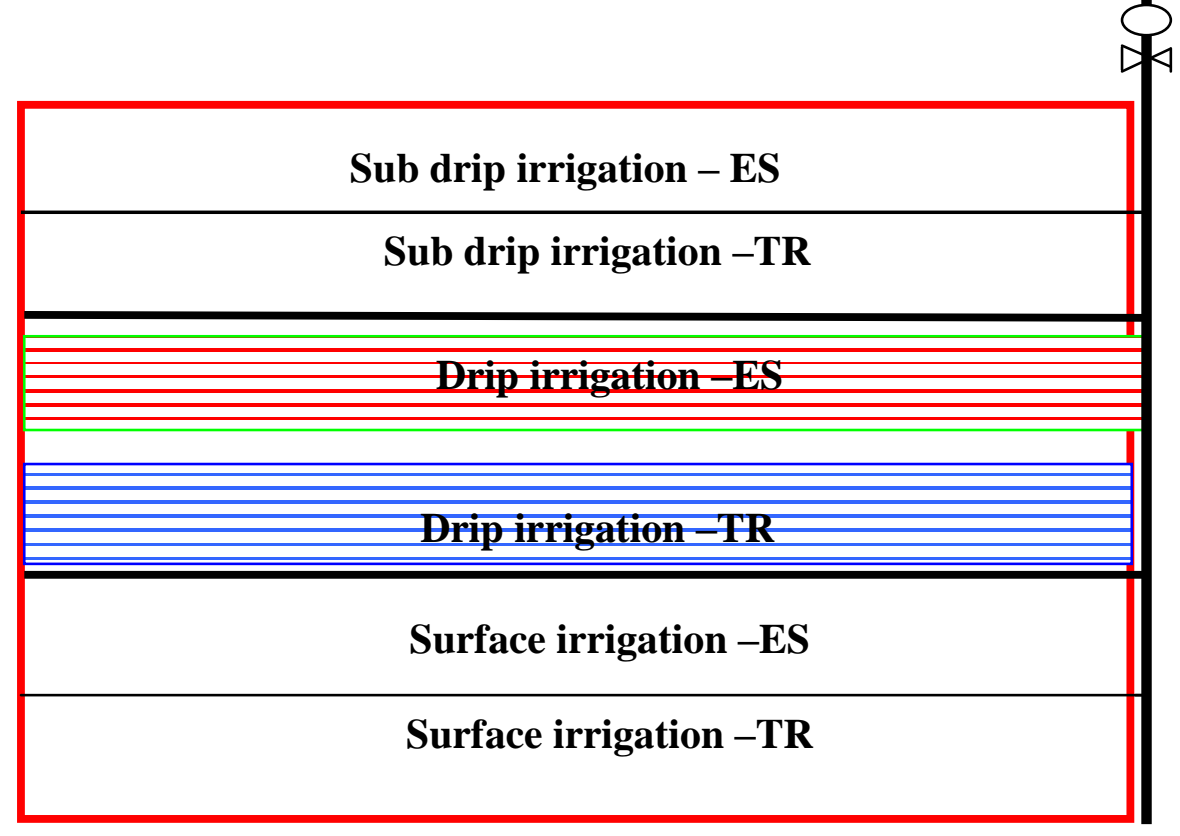

Fig (1): Layout of the experimental plots. 


\subsection{Crop biological properties}

Each experimental plot consisted of 9 lines and was represented by 12 random locations. Plant growth parameters were measured in each of the chosen locations. The investigated growth parameters were plant hight, root depth, plant weight, number of leaves, chlorophell percentage by chlorophell meter and stem weight

\subsection{Crop chemical properties:}

After measuring the biological properties, plant leaves were dried and analysed for O.C \%, O.M \%, Ash \%, T.N \% and C/N ratio.

\section{RESULTS AND DISCUSSION}

\section{3-1 The "OA-Fertigation" program}

The new software program, designed for fertigation in different locations depending on the expert system "ES", was given the name "OAFertigation Program",. This system consists of user interface, concepts, data bases and rules (Farag, 2011).

\section{3-1-1 The userinterface}

This consists of menu bar comprising three menus. The first one is start menu: consisting of three orders (open database, run and fertilizer selection). The second menu bar consists of several orders i.e. (climate database, soil database, water database, farm database, fertilizer database, manure database, crop database and irrigation system database) and the third menu is information about the program, as shown in Fig. (2).

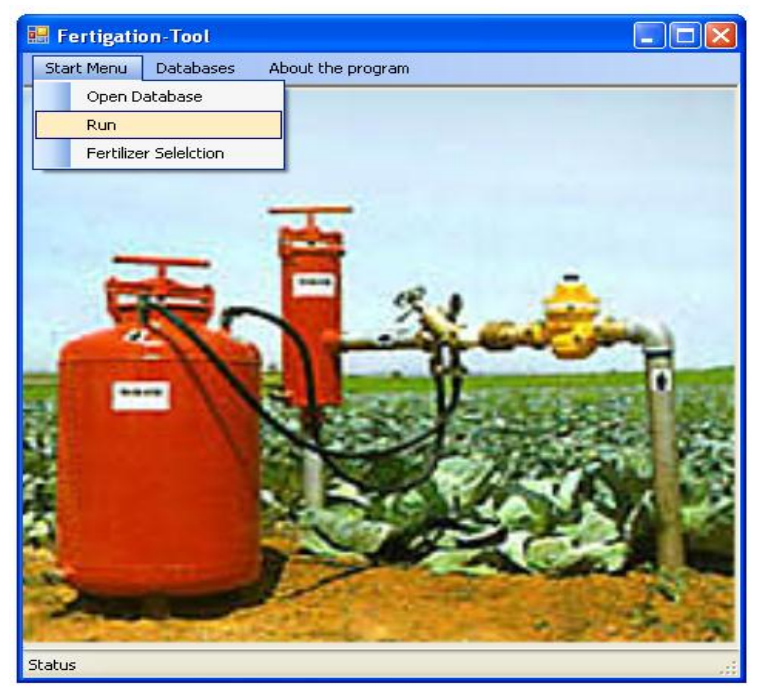

Fig (2): The program user interface. 


\section{3-2 Outputs of OA-Fertigation program and CROPWAT program}

3-2-1 Calculation of Eto in Kaliobia governorate according to different methods

Table (4) shows the evapotanspiration reference $\left(\mathrm{Et}_{\mathrm{o}}\right)$ calculated by OAFertigation program, CROPWAT and CLAC.

Table (4): Comparison between $\mathrm{Et}_{\mathrm{o}}$ calculation from CROPWAT model and irrigation model

\begin{tabular}{|c|c|c|c|c|}
\hline Month & $\begin{array}{c}\mathrm{Et}_{\mathrm{o}} \text { Model } \\
\mathrm{BM}\end{array}$ & $\begin{array}{c}\mathrm{Et}_{\mathrm{o}} \\
\mathrm{HG}^{* *}\end{array}$ & $\begin{array}{c}\mathrm{Et}_{\mathrm{o}} \\
\mathrm{CROPWAT}^{* * *}\end{array}$ & $\begin{array}{c}\text { From } \\
\text { CLAC**** }\end{array}$ \\
\hline $\mathbf{1}$ & 1.537 & 1.86 & 1.91 & 1.5 \\
\hline $\mathbf{2}$ & 1.764 & 2.71 & 2.33 & 2.1 \\
\hline $\mathbf{3}$ & 2.583 & 3.74 & 3.25 & 3.38 \\
\hline $\mathbf{4}$ & 3.747 & 5.01 & 4.85 & 3.2 \\
\hline $\mathbf{5}$ & 4.43 & 6.05 & 6.52 & 4.6 \\
\hline $\mathbf{6}$ & 4.969 & 6.33 & 6.78 & 6.7 \\
\hline $\mathbf{7}$ & 4.97 & 6.34 & 6.6 & 6.2 \\
\hline $\mathbf{8}$ & 4.646 & 5.98 & 6.21 & 5.9 \\
\hline $\mathbf{9}$ & 4.112 & 4.91 & 5.1 & 4.9 \\
\hline $\mathbf{1 0}$ & 3.375 & 3.89 & 4.1 & 3.7 \\
\hline $\mathbf{1 1}$ & 2.642 & 2.88 & 2.81 & 3.2 \\
\hline $\mathbf{1 2}$ & 1.604 & 1.90 & 1.96 & 1.2 \\
\hline
\end{tabular}

*Evapotranspriation calculated by OA-Fertigation program according to the equation of Penmann Montieth for open field and low tunnel.

** Evapotranspriation calculated by OA-Fertigation program according to the equation of Hargrivis for high tunnel.

*** Evapotranspriation calculated by “CROPWAT program".

**** Evapotranspriation measuerd by Center Laboratory of Agricultural Climate (CLAC). 


\section{3-2-2 Water requirement}

Table (5-a) shows the data required for irrigatin scheduling as outputs of the "CROPWAT" program under drip and sub-drip irrigation systems.

Table (5-a): Water requirement for bean under drip and sub-drip irrigation systems from the "CROPWAT program"

\begin{tabular}{|c|c|c|c|c|c|c|c|}
\hline Date & Day & Stage & Depl & dn & dg & $\mathbf{T}$ & II \\
\hline & & & $\%$ & $\mathbf{m m}$ & $\mathbf{m m}$ & (min) & days \\
\hline 5-Mar & 1 & Init & 57 & 16.8 & 18.7 & 50 & - \\
\hline 12-Mar & 8 & Init & 51 & 18.3 & 20.4 & 55 & 7 \\
\hline 20-Mar & 16 & Init & 50 & 22 & 24.5 & 66 & 8 \\
\hline 28-Mar & 24 & Dev & 54 & 27.5 & 30.6 & 83 & 8 \\
\hline 4-Apr & 31 & Dev & 57 & 32.8 & 36.4 & 98 & 7 \\
\hline 10-Apr & 37 & Dev & 53 & 33.7 & 37.4 & 101 & 6 \\
\hline 14-Apr & 41 & Dev & 48 & 32.6 & 36.2 & 98 & 4 \\
\hline 19-Apr & 46 & Dev & 56 & 40.7 & 45.2 & 122 & 5 \\
\hline 23-Apr & 50 & Dev & 50 & 38.3 & 42.5 & 115 & 4 \\
\hline 27-Apr & 54 & Mid & 51 & 38.9 & 43.2 & 117 & 4 \\
\hline 1-May & 58 & Mid & 52 & 39.6 & 44 & 119 & 4 \\
\hline 5-May & 62 & Mid & 55 & 41.9 & 46.5 & 126 & 4 \\
\hline 9-May & 66 & Mid & 55 & 41.9 & 46.5 & 126 & 4 \\
\hline 13-May & 70 & Mid & 58 & 43.8 & 48.6 & 131 & 4 \\
\hline 17-May & 74 & Mid & 58 & 44.4 & 49.3 & 133 & 4 \\
\hline 21-May & 78 & Mid & 59 & 44.5 & 49.5 & 134 & 4 \\
\hline 25-May & 82 & Mid & 59 & 45 & 50 & 135 & 4 \\
\hline 29-May & 86 & Mid & 59 & 45 & 50 & 135 & 4 \\
\hline 2-Jun & 90 & Mid & 56 & 42.5 & 47.2 & 127 & 4 \\
\hline 6-Jun & 94 & End & 53 & 39.9 & 44.3 & 120 & 4 \\
\hline 10-Jun & 98 & End & 53 & 39.9 & 44.3 & 120 & 4 \\
\hline 17-Jun & 105 & End & 56 & 42.5 & 47.2 & 127 & 7 \\
\hline 22-Jun & End & End & 29 & & & & \\
\hline Total & & & & & 902.5 & & \\
\hline
\end{tabular}

- $\quad$ * Irrigation intervals

- $\quad$ ** Irrigation of net water requirement depth

- $\quad * * *$ Irrigation of growth water requirement depth 
Table (5-b) shows the data required for irrigatin scheduling as outputs of the "CROPWAT" program under furrow irrigation

Table (5-b): Water requirement for bean under furrow irrigation system from CROPWAT program

\begin{tabular}{|c|c|c|c|c|c|c|c|c|}
\hline Date & Day & Stage & Depl & $\mathbf{d n}$ & $\mathbf{d g}$ & Tn & Tco & II \\
\hline & & & $\mathbf{\%}$ & $\mathbf{m m}$ & $\mathbf{m m}$ & $\mathbf{m i n}$ & & \\
\hline 5-Mar & 1 & Init & 57 & 16.8 & 28 & 8.4 & 15 & - \\
\hline 12-Mar & 8 & Init & 51 & 18.3 & 30.6 & 9.18 & 16 & 7 \\
\hline 20-Mar & 16 & Init & 50 & 22 & 36.7 & 11.01 & 18 & 8 \\
\hline 28-Mar & 24 & Dev & 54 & 27.5 & 45.9 & 13.77 & 21 & 8 \\
\hline 4-Apr & 31 & Dev & 57 & 32.8 & 54.7 & 16.41 & 23 & 7 \\
\hline 10-Apr & 37 & Dev & 53 & 33.7 & 56.2 & 16.86 & 24 & 6 \\
\hline 14-Apr & 41 & Dev & 48 & 32.6 & 54.3 & 16.29 & 23 & 4 \\
\hline 19-Apr & 46 & Dev & 56 & 40.7 & 67.8 & 20.34 & 27 & 5 \\
\hline 23-Apr & 50 & Dev & 50 & 38.3 & 63.8 & 19.14 & 26 & 4 \\
\hline 27-Apr & 54 & Mid & 51 & 38.9 & 64.8 & 19.44 & 26 & 4 \\
\hline 1-May & 58 & Mid & 52 & 39.6 & 66.1 & 19.83 & 27 & 4 \\
\hline 5-May & 62 & Mid & 55 & 41.9 & 69.8 & 20.94 & 28 & 4 \\
\hline 9-May & 66 & Mid & 55 & 41.9 & 69.8 & 20.94 & 28 & 4 \\
\hline 13-May & 70 & Mid & 58 & 43.8 & 72.9 & 21.87 & 29 & 4 \\
\hline 17-May & 74 & Mid & 58 & 44.4 & 74 & 22.2 & 29 & 4 \\
\hline 21-May & 78 & Mid & 59 & 44.5 & 74.2 & 22.26 & 29 & 4 \\
\hline 25-May & 82 & Mid & 59 & 45 & 75 & 22.5 & 30 & 4 \\
\hline 29-May & 86 & Mid & 59 & 45 & 75 & 22.5 & 30 & 4 \\
\hline 2-Jun & 90 & Mid & 56 & 42.5 & 70.8 & 21.24 & 28 & 4 \\
\hline 6-Jun & 94 & End & 53 & 39.9 & 66.5 & 19.95 & 27 & 4 \\
\hline 10-Jun & 98 & End & 53 & 39.9 & 66.5 & 19.95 & 27 & 4 \\
\hline 17-Jun & 105 & End & 56 & 42.5 & 70.8 & 21.24 & 28 & 7 \\
\hline 22-Jun & End & End & 29 & & & & & \\
\hline Total & & & & & $\mathbf{1 3 5 4 . 2}$ & & $\mathbf{5 6 8 7 . 6 4} \mathbf{3}$ /f \\
\hline
\end{tabular}

See footnotes of Table (5-a) 
Table (6-a) shows the data required for irrigatin scheduling as outputs of the "OA-Fertigation" program under drip and sub drip irrigation systems.

Table (6-a): Water requirement for bean under drip and sub drip irrigation systems as outputs of OA-Fertigation program

\begin{tabular}{|c|c|c|c|c|c|c|c|}
\hline $\begin{array}{c}\text { Irrigation } \\
\text { date }\end{array}$ & $\begin{array}{c}\text { Root } \\
\mathbf{d e p t h} \\
(\mathbf{c m})\end{array}$ & $\begin{array}{c}\text { Etc } \\
(\mathbf{m m})\end{array}$ & $\begin{array}{c}\mathbf{d n} \\
(\mathbf{c m})\end{array}$ & $\begin{array}{c}\mathbf{d g} \\
\mathbf{( c m})\end{array}$ & $\begin{array}{c}\text { Irrigation } \\
\text { time (min) }\end{array}$ & WR $^{* *}$ & II $^{*}$ \\
\hline $3 / 5 / 2010$ & 15 & 0.73 & 0.77 & 0.96 & 26 & 2.17 & - \\
\hline $3 / 16 / 2010$ & 15 & 0.73 & 0.77 & 0.96 & 26 & 2.17 & 11 \\
\hline $3 / 27 / 2010$ & 18.88 & 1.73 & 0.97 & 1.21 & 33 & 2.75 & 11 \\
\hline $4 / 2 / 2010$ & 26.23 & 2.28 & 1.35 & 1.68 & 45 & 3.75 & 6 \\
\hline $4 / 8 / 2010$ & 32.83 & 2.83 & 1.69 & 2.1 & 57 & 4.75 & 6 \\
\hline $4 / 14 / 2010$ & 37.8 & 3.38 & 1.94 & 2.41 & 65 & 5.42 & 6 \\
\hline $4 / 20 / 2010$ & 40 & 3.93 & 2.05 & 2.56 & 69 & 5.75 & 6 \\
\hline $4 / 25 / 2010$ & 40 & 5.71 & 2.05 & 2.56 & 69 & 5.75 & 5 \\
\hline $4 / 29 / 2010$ & 40 & 5.71 & 2.05 & 2.56 & 69 & 5.75 & 4 \\
\hline $5 / 3 / 2010$ & 40 & 5.71 & 2.05 & 2.56 & 69 & 5.75 & 4 \\
\hline $5 / 7 / 2010$ & 40 & 5.71 & 2.05 & 2.56 & 69 & 5.75 & 4 \\
\hline $5 / 11 / 2010$ & 40 & 5.71 & 2.05 & 2.56 & 69 & 5.75 & 4 \\
\hline $5 / 15 / 2010$ & 40 & 5.71 & 2.05 & 2.56 & 69 & 5.75 & 4 \\
\hline $5 / 19 / 2010$ & 40 & 5.71 & 2.05 & 2.56 & 69 & 5.75 & 4 \\
\hline $5 / 23 / 2010$ & 40 & 5.71 & 2.05 & 2.56 & 69 & 5.75 & 4 \\
\hline $5 / 27 / 2010$ & 40 & 5.71 & 2.05 & 2.56 & 69 & 5.75 & 4 \\
\hline $5 / 31 / 2010$ & 40 & 5.71 & 2.05 & 2.56 & 69 & 5.75 & 4 \\
\hline $6 / 4 / 2010$ & 40 & 5.32 & 2.05 & 2.56 & 69 & 5.75 & 4 \\
\hline $6 / 8 / 2010$ & 40 & 4.52 & 2.05 & 2.56 & 69 & 5.75 & 4 \\
\hline $6 / 13 / 2010$ & 40 & 3.53 & 2.05 & 2.56 & 69 & 5.75 & 5 \\
\hline $6 / 19 / 2010$ & 40 & 2.34 & 2.05 & 2.56 & 69 & 5.75 & - \\
\hline Total & & & & & & $\mathbf{1 0 7 . 2 6}$ & \\
\hline & & & $\mathbf{2 0 0 2 . 2} \mathbf{m}^{\mathbf{3} / \mathbf{f}}$ & & & \\
\hline & & & & & \\
\hline
\end{tabular}

- $\quad$ * Irrigation intervals, days.

- $\quad * *$ Water requirement, $\mathrm{m}^{3} / 225 \mathrm{~m}^{2} / \mathrm{II}$. 
Table (6-b) shows the data required for irrigatin scheduling as outputs of the "OA-Fertigation" program under furrow irrigation.

Table (6-b): Water requirement for bean under furrow irrigation systems as outputs of the "OA-Fertigation" program.

\begin{tabular}{|c|c|c|c|c|c|c|c|}
\hline $\begin{array}{c}\text { Irrigation } \\
\text { date }\end{array}$ & $\begin{array}{c}\text { Root } \\
\text { depth } \\
(\mathbf{c m})\end{array}$ & $\begin{array}{c}\text { Etc } \\
(\mathbf{m m})\end{array}$ & $\begin{array}{c}\mathbf{d n} \\
(\mathbf{c m})\end{array}$ & $\begin{array}{c}\mathbf{d g} \\
(\mathbf{c m})\end{array}$ & $\begin{array}{c}\text { Irrigation } \\
\text { time } \\
(\mathbf{m i n})\end{array}$ & WR & II \\
\hline $3 / 5 / 2010$ & 15 & 1.03 & 2.2 & 4.26 & 14 & 1.17 & - \\
\hline $3 / 26 / 2010$ & 17.65 & 1.69 & 2.59 & 5.01 & 15 & 1.25 & 21 \\
\hline $4 / 10 / 2010$ & 34.7 & 3.09 & 5.09 & 9.85 & 22 & 1.83 & 15 \\
\hline $4 / 26 / 2010$ & 40 & 5.71 & 5.87 & 11.35 & 25 & 2.08 & 16 \\
\hline $5 / 6 / 2010$ & 40 & 5.71 & 5.87 & 11.35 & 25 & 2.08 & 10 \\
\hline $5 / 16 / 2010$ & 40 & 5.71 & 5.87 & 11.35 & 25 & 2.08 & 10 \\
\hline $5 / 26 / 2010$ & 40 & 5.71 & 5.87 & 11.35 & 25 & 2.08 & 10 \\
\hline $6 / 5 / 2010$ & 40 & 5.12 & 5.87 & 11.35 & 25 & 2.08 & 10 \\
\hline $6 / 16 / 2010$ & 40 & 2.93 & 5.87 & 11.35 & 25 & 2.08 & - \\
\hline Total & & & & & $\mathbf{1 6 . 7 3}$ & $\mathbf{2 8 1 0 . 6 4} \mathbf{~ m}^{\mathbf{3}} / \mathbf{f}$ \\
\hline
\end{tabular}

\section{3-2-3 Nutrient requirements:}

Table (7): Fertilizer requirements for bean under drip and sub drip irrigation system (ppm) as outputs of OA-Fertigation program and the traditional method.

\begin{tabular}{|c|c|c|c|c|c|c|}
\hline \multirow{2}{*}{ Stage } & \multicolumn{2}{|c|}{$\begin{array}{c}\text { Ammonium } \\
\text { nitrate } \\
\text { (ppm) }\end{array}$} & \multicolumn{2}{c|}{$\begin{array}{c}\text { Phosphoric } \\
\text { acid(ppm) }\end{array}$} & \multicolumn{2}{c|}{$\begin{array}{c}\text { Potassium } \\
\text { sulfate(ppm) }\end{array}$} \\
\cline { 2 - 7 } & ES & TR & ES & TR & ES & TR \\
\hline S1 & 65 & 161 & 44 & 120 & 0 & 200 \\
\hline S2 & 84 & 250 & 36 & 100 & 0 & 230 \\
\hline S3 & 57 & 170 & 29 & 80 & 0 & 300 \\
\hline Tank & \multicolumn{2}{|c|}{ A or B } & \multicolumn{2}{|c|}{ A } & \multicolumn{2}{|c|}{ A or B } \\
\hline
\end{tabular}

- $\mathbf{S} 1=$ From beginning of seedling emergence up to beginning of flowering

- S2= From beginning of flowering up to beginning of harvesting

- S3= From beginning of harvesting up to one week before end of harvesting 
- $\mathrm{TR}=$ Traditional method (CROPWAT program was used for the scheduling of the irrigation together with the traditional methods of fertigation as outlined by the Ministry of Agriculture, Egypt).

- $\mathrm{ES}=\mathrm{OA}-$ Fertigation program.

Table (8): Fertilizer requirements for bean under drip and sub drip irrigation (g / week) as outputs of OA-Fertigation program.

\begin{tabular}{|c|c|c|c|c|c|c|}
\hline Stage & \multicolumn{2}{|c|}{$\begin{array}{c}\text { Ammonium Nitrate } \\
\text { (g/week) }\end{array}$} & \multicolumn{2}{|c|}{$\begin{array}{c}\text { Phosphoric acid } \\
\left(\mathbf{c m}^{3} / \text { week }\right)\end{array}$} & \multicolumn{2}{c|}{$\begin{array}{c}\text { Potassium } \\
\text { sulfate } \\
\text { (g/week) }\end{array}$} \\
\hline & ES & TR & ES & TR & ES & TR \\
\hline S1 & 445 & 2080 & 185 & 843 & 0 & 2584 \\
\hline S2 & 664 & 3231 & 155 & 702 & 0 & 2972 \\
\hline S3 & 510 & 2197 & 124 & 562 & 0 & 3877 \\
\hline Tank & \multicolumn{2}{|c|}{ A or B } & \multicolumn{2}{|c|}{ A } & \multicolumn{2}{|c|}{ A or B } \\
\hline
\end{tabular}

Table (9): Fertilizer requirements for bean under furrow irrigation (g / week) as outputs of OA-Fertigation program

\begin{tabular}{|c|c|c|c|c|c|c|}
\hline Stage & \multicolumn{2}{|c|}{$\begin{array}{c}\text { Ammonium Nitrate } \\
\text { (g /week) }\end{array}$} & \multicolumn{2}{c|}{$\begin{array}{c}\text { Phosphoric acid } \\
\left(\mathbf{c m}^{\mathbf{3}} \text { / week) }\right.\end{array}$} & $\begin{array}{c}\text { Potassium sulfate } \\
\text { (g/week) }\end{array}$ \\
\hline & ES & TR & ES & TR & ES & TR \\
\hline S1 & 648 & 3122 & 342 & 1265 & 0 & 3878 \\
\hline S2 & 972 & 4847 & 288 & 1054 & 0 & 4460 \\
\hline S3 & 747 & 3296 & 225 & 843 & 0 & 5817 \\
\hline Tank & \multicolumn{2}{|c|}{ A or B } & \multicolumn{2}{|c|}{ A } & \multicolumn{2}{c|}{ A or B } \\
\hline
\end{tabular}

\section{3-3 Biological properties}

Data presented in Table (10) illustrate values of the growth prarameters of the bean crop achieved by the designed fertigation system (ES) and Traditional fertigation system (TR). 
Table (10): The biological properties for bean crop under the ES and TR systems

\begin{tabular}{|l|l|c|c|c|c|c|c|}
\hline \multirow{2}{*}{ prameters } & \multirow{2}{*}{ Units } & \multicolumn{7}{|c|}{ Irrigation system } \\
\cline { 3 - 8 } & & \multicolumn{2}{|c|}{ SD } & \multicolumn{2}{|c|}{ DR } & \multicolumn{2}{|c|}{ FR } \\
\cline { 3 - 8 } & & ES & TR & ES & TR & ES & TR \\
\hline Plant Height & $\mathbf{c m}$ & 32.8 & 31.8 & 30.7 & 27.5 & 37.2 & 34.1 \\
\hline Root mass & $\mathbf{g}$ & 1.7 & 1.4 & 1.3 & 1.1 & 1.9 & 1.3 \\
\hline Root depth & $\mathbf{~ c m}$ & 23.9 & 22.9 & 20.5 & 18.2 & 26.9 & 25.7 \\
\hline Plant mass & $\mathbf{g}$ & 31.5 & 30.2 & 25.8 & 22.3 & 60.7 & 36.3 \\
\hline Chlorophyll & \% & 47.7 & 46.4 & 45.8 & 41.1 & 41.9 & 41 \\
\hline Stem mass & $\mathbf{g}$ & 12.7 & 10.7 & 17.7 & 6.4 & 21.8 & 13.1 \\
\hline $\begin{array}{l}\text { Number of } \\
\text { leaves }\end{array}$ & & & & & & & \\
\hline
\end{tabular}

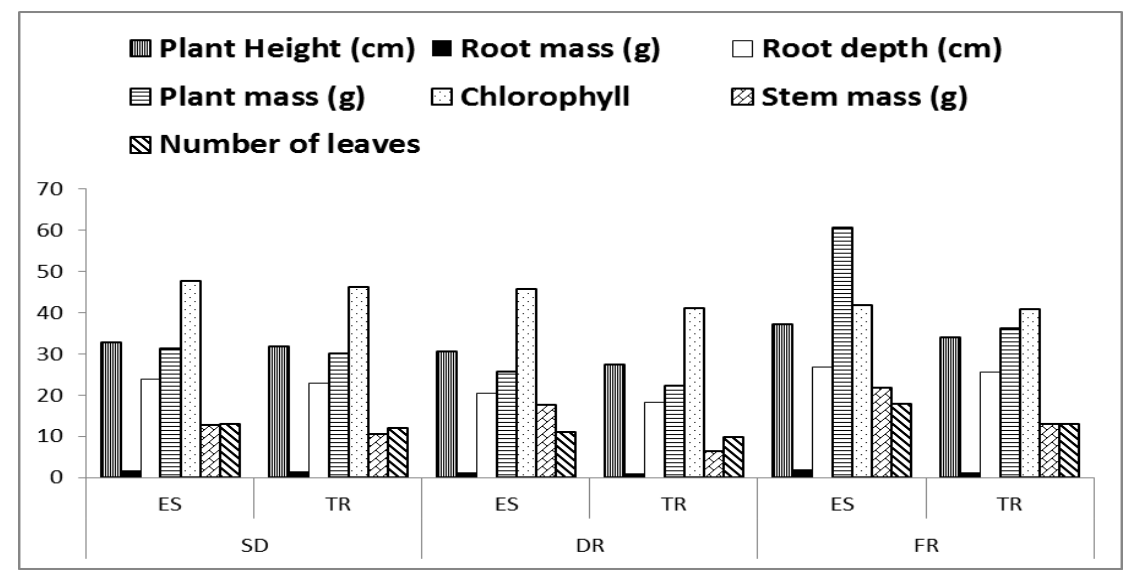

Fig (3): The biological properties for bean crop under the ES and TR systems.

- $\mathrm{SD}=$ sub drip irrigation

- $\mathrm{DR}=$ drip irrigation

- $\mathrm{FR}=$ furrow irrigation

It is obvions from Tables (10, 11, 12 and 13) and Fig. (3) That values of all the studied growth parameters, yield and its components achiveved due to the fertigation management according to the OA-Fertigation program were obviously higher than the corresponding ones from the 
traditional fertigation system. Such a finding indicates the superiority of the "OA-Fertigation" program as a tool, which means more accurate and fast knowledge for better management of irrigation and fertilization.

\section{3-4 Chemical properties}

\subsubsection{Plant analysis}

We have taken 12 plants from each replicate, and the chemical analyses are as shown in Table (11).

Table (11): Bean crop analysis

\begin{tabular}{|l|l|c|c|c|c|c|}
\hline $\begin{array}{l}\text { Irrigation } \\
\text { systems }\end{array}$ & $\begin{array}{c}\text { Fertigation } \\
\text { system }\end{array}$ & O.C \% & $\begin{array}{c}\text { O.M } \\
\mathbf{\%}\end{array}$ & $\begin{array}{c}\text { Ash } \\
\mathbf{\%}\end{array}$ & $\begin{array}{c}\text { T.N } \\
\mathbf{\%}\end{array}$ & $\begin{array}{c}\text { C/N } \\
\text { Ratio }\end{array}$ \\
\hline $\begin{array}{l}\text { Sub Drip } \\
\text { Irrigation }\end{array}$ & ES & 45.8 & 79 & 21 & 5.38 & 8.5 \\
\cline { 2 - 7 } $\begin{array}{l}\text { Drip } \\
\text { Irrigation }\end{array}$ & ES & 47 & 81 & 19 & 5.4 & 8.8 \\
\hline Furrow & ER & 47.1 & 81.3 & 18.8 & 5.21 & 9 \\
\cline { 2 - 8 } Irrigation & TR & 47.3 & 81.5 & 18.5 & 4.76 & 9.9 \\
\hline
\end{tabular}

\section{$\underline{\text { 3-5 Mass of } 100 \text { seeds }}$}

Data presented in Table (12) illustrate values of 100 seed mass of bean.

Table (12): Mass of 100 seeds

\begin{tabular}{|c|c|c|c|c|c|}
\hline \multicolumn{6}{|c|}{ Mass of 100 seeds $(\mathrm{g})$} \\
\hline $\begin{array}{l}\text { Irrigation } \\
\text { systems }\end{array}$ & $\begin{array}{l}\text { Fertigation } \\
\text { system }\end{array}$ & R1 & $\mathbf{R 2}$ & $\mathbf{R 3}$ & Mean \\
\hline \multirow{2}{*}{$\begin{array}{l}\text { Sub Drip } \\
\text { Irrigation }\end{array}$} & ES & 37 & 36 & 38 & 37 \\
\hline & TR & 33 & 32 & 34 & 33 \\
\hline \multirow{2}{*}{$\begin{array}{l}\text { Drip } \\
\text { Irrigation }\end{array}$} & ES & 39 & 40 & 38 & 39 \\
\hline & TR & 38 & 37 & 36 & 38 \\
\hline \multirow{2}{*}{$\begin{array}{l}\text { Furrow } \\
\text { Irrigation }\end{array}$} & ES & 43 & 41 & 42 & 43 \\
\hline & TR & 42 & 40 & 41 & 42 \\
\hline
\end{tabular}




\section{3-6 Seed dry-yield}

Data presented in Table (13) illustrate values of seed dry-yield of bean.

Table (13): Seed dry-yield

\begin{tabular}{|c|c|c|c|c|c|}
\hline \multicolumn{7}{|c|}{ Seed dry-yield (kg / f) } \\
\hline $\begin{array}{c}\text { Irrigation } \\
\text { systems }\end{array}$ & $\begin{array}{c}\text { Fertigation } \\
\text { system }\end{array}$ & $\mathbf{R 1}$ & $\mathbf{R 2}$ & $\mathbf{R 3}$ & Mean \\
\hline $\begin{array}{c}\text { Sub Drip } \\
\text { Irrigation }\end{array}$ & $\mathbf{E S}$ & 1857.1 & 1764.3 & 1860.9 & 1827.4 \\
\cline { 2 - 6 } & $\mathbf{T R}$ & 1095.7 & 797.3 & 1690.0 & 1194.3 \\
\hline $\begin{array}{c}\text { Drip } \\
\text { Irrigation }\end{array}$ & $\mathbf{E S}$ & 1329.7 & 1300.0 & 1314.9 & 1314.9 \\
\cline { 2 - 6 } & $\mathbf{T R}$ & 1113.0 & 1105.6 & 1109.3 & 1109.3 \\
\hline $\begin{array}{c}\text { Furrow } \\
\text { Irrigation }\end{array}$ & $\mathbf{E S}$ & 2864 & 2805 & 2923 & 2864.0 \\
\cline { 2 - 6 } & $\mathbf{T R}$ & 2564 & 2500 & 2628 & 2564.0 \\
\hline
\end{tabular}

3-7 Water Use Efficiency (WUE) and Nutrient Use Efficiency (NUE)

Data in Table (14) reveal that values of. WUE, as well as those of NUE, under the "OA-Fertigation" program were higher than the corresponding ones achieved under the traditional method. Accordingly, we can deduce that the "OA-Fertigation" program resulted in higher yield of bean crop than the traditional method and, at the same time, could provide better management for both irrigation and fertilization as noticed from the values of both WUE and NUE.

Table (14): WUE and NUE for bean crop

\begin{tabular}{|c|c|c|c|}
\hline \multirow{2}{*}{$\begin{array}{c}\text { Irrigation } \\
\text { systems }\end{array}$} & \multirow{2}{*}{$\begin{array}{c}\text { Fertigation } \\
\text { system }\end{array}$} & \multicolumn{2}{|c|}{ Bean } \\
\hline & & $\begin{array}{c}\text { WUE } \\
\text { Kg. } \mathbf{~ m}^{-3}\end{array}$ & $\begin{array}{c}\text { NUE } \\
\text { Kg. } \text { Kg }^{-1}\end{array}$ \\
\hline \multirow{2}{*}{$\begin{array}{l}\text { Sub Drip } \\
\text { Irrigation }\end{array}$} & ES & 0.91 & 13.4 \\
\hline & TR & 0.32 & 8.8 \\
\hline \multirow{2}{*}{$\begin{array}{l}\text { Drip } \\
\text { Irrigation }\end{array}$} & ES & 0.66 & 9.7 \\
\hline & TR & 0.29 & 8.2 \\
\hline \multirow{2}{*}{$\begin{array}{l}\text { Furrow } \\
\text { Irrigation }\end{array}$} & ES & 1.02 & 21.1 \\
\hline & TR & 0.45 & 18.9 \\
\hline
\end{tabular}

\section{REFERENCES:}

Awady, M. N., 2010, Computer applications in Agricultutal Engineering, Txt Bk., Ch. 7 on Expert systems and their use in Agricultural Engineering, Ag. Eng. Dept., Col. Ag., Al Azhar U.: 160 p. (in Arabic). 
Charles M., 2007, Fertigation chemicals, FERTIGATION. 1995. Burt, C.M., K. O'Connor, and T. Ruehr. ISBN 0-9643634-1-0. 295 p.

Dent, J. B. and J. W. Jones, 1989, Advantages of the expert systems, J. Agric. Sys., 31(1): 150-155.

Farag, A. A., 2011, Fertigation Technique Management Based on Expert System, Dept. Agri. Eng. Fac. Agrc. Benha Univ., Egypt.

FAO, 2005, Fertilizer use by crop in Egypt, First version, published by FAO, Rome. 57p.

FAO/RNEA, 1992, Wastewater as a crop nutrient source. Tech. Bul. No. 5. , p: 15

Kabany, A. G., 2003, Uses of expert systems in agricultural engineering, Unpub Review article, Fac. of Agric., Ain Shams Univ., Egypt.

Refea, A. A., 1998, Egyptian research program for developing expert systems in agriculture, $7^{\text {th }}$ Int. Conf. on Comp. in Agric., 20-26 July, Orlando, FL, USA.

Victoria, 2010, Critical soil test values and pasture response, Dept. of primary industries, Agriculter, june2010 edition.

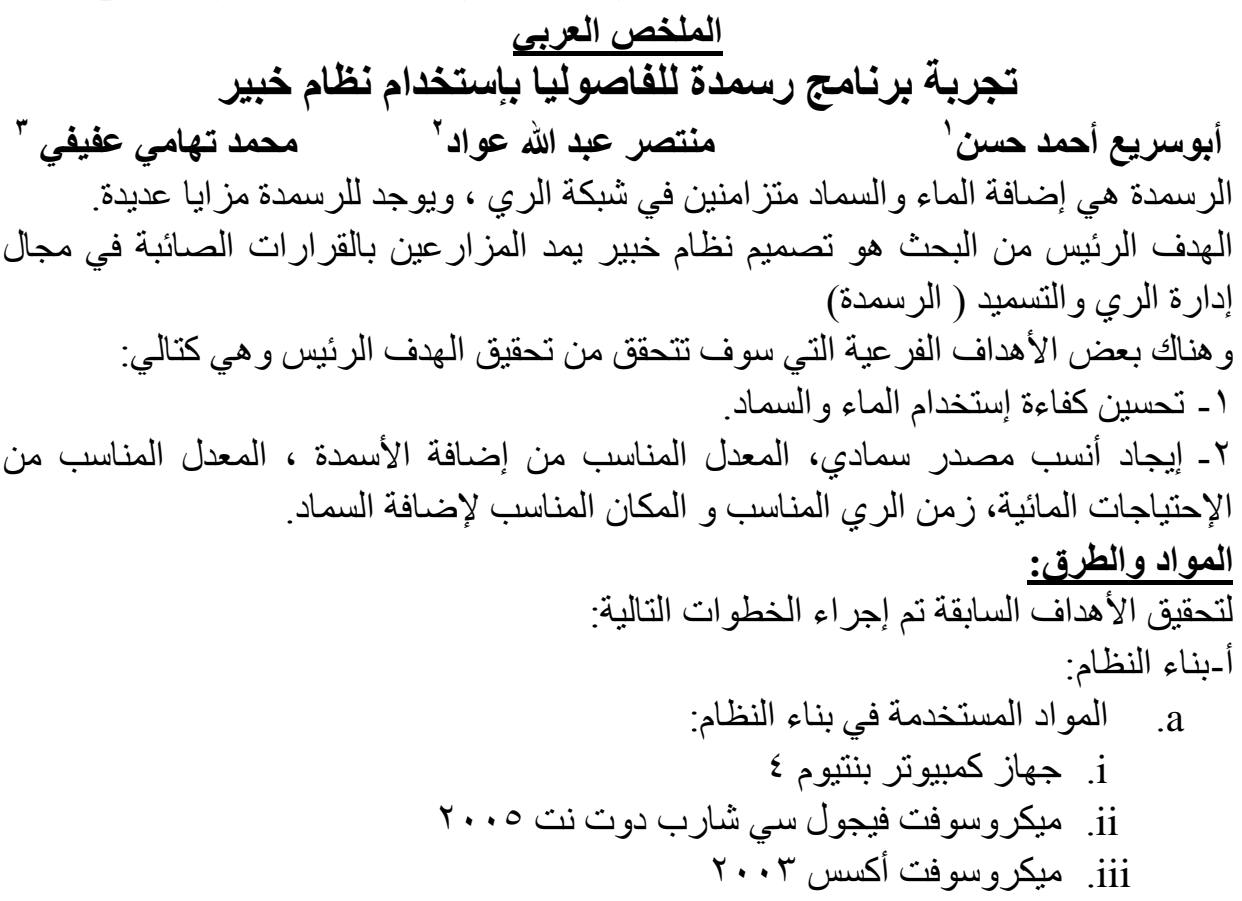

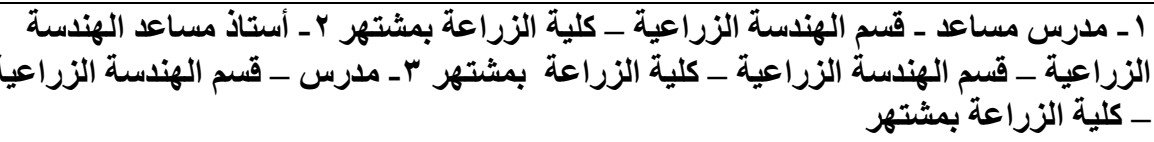




$$
\begin{aligned}
& \text { b الطرق المتبعة لبناء النظام: } \\
& \text { i.تعريف المشكلة: }
\end{aligned}
$$

المشكلة التي يدرسها هذا البحث هي إيجاد تقنية جديده تستخدم في حل المشاكل

$$
\text { المتعلقة بإدارة الرسمدة. }
$$

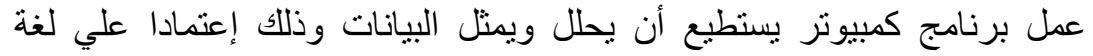

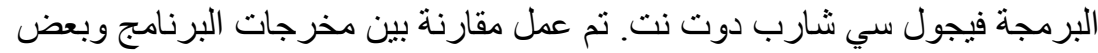

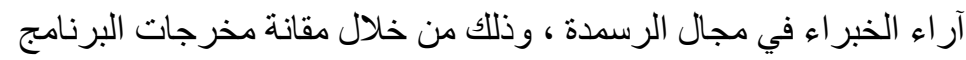

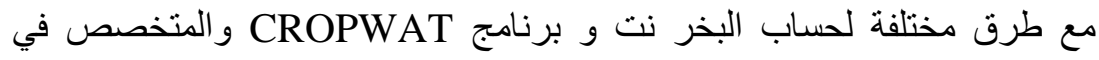

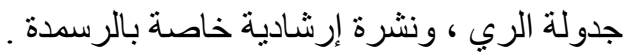

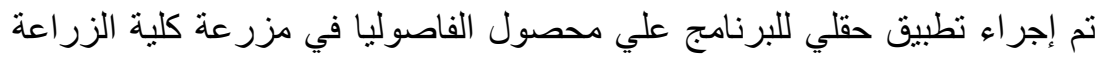
بمشتهر.

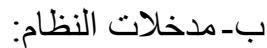
بيانات التربة - بيانات المناخ - بيانات الماء - بيانات الأسمدة - بيانات المحصول- بيانات

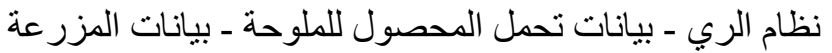

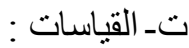

تم تقسيم التجربة إلي ست قطاعات ، القطعة الأولي والثانبة بها نظام الري بالتقفيط تحت

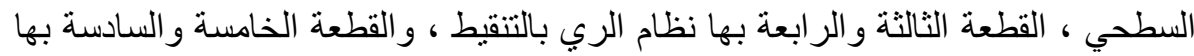

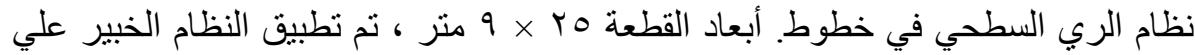
القطع الأولي، الثالثة و الر ابعة ، والقطع الآخرى تم تطبيق الطريقة التقليدية في باقي القطع. a a

كل قطعة تجريبية تتكون من 9 خطوط ، تم أخذ ب ا ع عينة نباتية من كل قطعة تجريبية

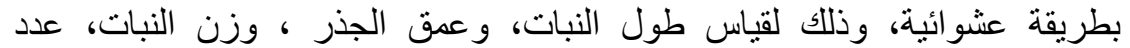
الأوراق، نسبة الكلوروفيل، وزن النة، وذئن الساق.

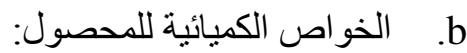

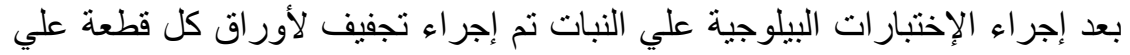

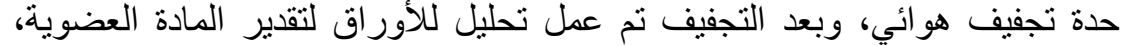

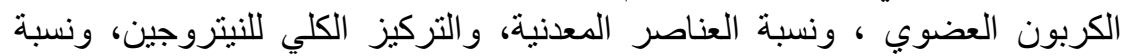

$$
\begin{aligned}
& \text { الكربون إلي النيتروجين. } \\
& \text { أوضحت أهم النتائج ما يلي : }
\end{aligned}
$$

1 ـ تفوق النظام الخبير في سر عة وسهولة إستخدامعه عن الطريقة التقليدية.

r- أوضحت در اسة الخو اص البيولوجية و الكيميائية تفوق النظام الخبير عن الطريقة التقليدية. r- زيادة إنتاج المحصول تحت النظام الخبير عنه للطريقة التقليدية تحت نظم الري الثنلاثة

(الري بالتنقيط تحت السطحي، الري بالتنقيط ، الري الرئ السطحي في خطوط).

ع - زيادة وزن . احابة تحت النظام الخبير عنه تحت الطريقة التقليدية. هـ زيادة كفاءة إستخدام المياه و السماد تحت النظام الخبير عنه في حالة الطريقة التقليدية. 